\title{
Abundância e sazonalidade das espécies de Leptoceridae (Insecta, Trichoptera) capturadas com armadilha luminosa no Estado do Paraná, Brasil ${ }^{1}$
}

\author{
Gisele Luziane de Almeida 2, 3 \\ Luciane Marinoni ${ }^{2,4}$
}

\begin{abstract}
Abundance and seasonality of Leptoceridae species (Insecta, Trichoptera) captured with light trap at Paraná State, Brazil. From 126,340 adult specimens of Trichoptera captured with light trap during one year of the project called "Survey of the Entomological Fauna in Paraná State" (Paraná, Brazil), 6,375 belong to the family Leptoceridae. The individuals of the family were identified and a list of species is presented. The following species are recorded for the first time to Paraná State: Achoropsyche duodecimpunctata (Návas, 1916); Nectopsyche aureovittata Flint, 1983; Nectopsyche fuscomaculata Flint, 1983; Nectopsyche muhni (Návas, 1916); Nectopsyche separata (Banks, 1920); Nectopsyche ortizi Holzenthal, 1995; Nectopsyche splendida (Návas, 1917) and Triplectides gracilis (Burmeister, 1839). Discussion on individual abundance and species seasonality are also presented. KEY WORDS. Trichoptera, Leptoceridae, abundance, seasonality, species richness, light trap, Paraná, Brazil
\end{abstract}

Este trabalho faz parte de uma série de contribuições realizada com material entomológico proveniente de coletas no estado do Paraná, de agosto de 1986 a julho de 1988. Neste período, em um projeto denominado "Projeto de Levantamento da Fauna Entomológica no Estado do Paraná" (PROFAUPAR), representantes de vários grupos de insetos foram capturados com auxílio de armadilhas luminosa e Malaise. Oito localidades do Estado foram amostradas, cada uma delas com características geomorfológicas, climáticas e florísticas detalhadas em MARINONI \& DUTRA (1993).

Um significativo número de insetos pertencentes à Ordem Trichoptera foi capturado durante o primeiro ano de realização do projeto (agosto/1986 a julho/1987). Visto que, grande parte dos tricópteros tem comportamento crepuscular e noturno, a maioria absoluta foi capturada com armadilha luminosa: 126.340 exemplares em um total de 126.641 tricópteros.

Dentre as famílias de Trichoptera com distribuição neotropical, a família Hydropsychidae foi a primeira a ser estudada com o material advindo das coletas do PROFAUPAR. A abundância e a sazonalidade das espécies do primeiro ano de coleta são apresentadas e discutidas em MARINONI \& ALMEIDA (2000).

1) Contribuição número 1211 do Departamento de Zoologia, Universidade Federal do Paraná.

2) Departamento de Zoologia, Universidade Federal do Paraná. Caixa Postal 19020, 81531-990 Curitiba, Paraná, Brasil.

3) Bolsista da CAPES. E-mail: gisele@bio.ufpr.br

4) E-mail: Imarinon@bio.ufpr.br 
A família Leptoceridae, também um grande grupo dentro da Ordem, apresenta distribuição geográfica ampla. São conhecidos representantes em todas as regiões do mundo, sendo uma das famílias dominantes na região Neotropical. É dividida em duas subfamílias: Triplectidinae, com três tribos e Leptocerinae, com oito. Para o Brasil são conhecidas 39 espécies em oito gêneros (Ralph W. Holzenthal, comunicação pessoal).

Como característica marcante para a família, as larvas podem habitar qualquer ambiente de água doce: desde poças temporárias a grandes rios de água corrente, inclusive com quedas d'água (FLINT 1983, 1991b; WIGGINS 1996). Devido à sensibilidade que algumas espécies apresentam em relação às modificações ambientais, são consideradas boas bioindicadoras de diferentes níveis de poluição em corpos d'água (ANGRISANO 1995; PÉREZ 1988; WigGINS 1996).

A plasticidade de adaptação das larvas de Leptoceridae é provavelmente devido a diversidade da familia o que a torna de especial interesse, pois podem apresentar um grande potencial para o repovoamento de áreas degradadas.

O conhecimento da Ordem no Paraná e, conseqüentemente da região Neotropical, é o principal objetivo deste trabalho, tendo em vista que pouco se conhece sobre a fauna de tricópteros desta região. Como objetivos mais específicos são analisadas a abundância e a sazonalidade das espécies de Leptoceridae, capturadas com armadilha luminosa no Estado durante o primeiro ano do projeto.

\section{MATERIAL E MÉTODOS}

As áreas de estudo e os métodos de amostragem estão detalhados em MARINONI \& DUTRA (1993) e MARINONI \& ALMEIDA (2000).

Os insetos capturados foram preservados em álcool hidratado a $70 \% \mathrm{e}$ encontram-se depositados na Coleção de Entomologia Pe. Jesus S. Moure, Departamento de Zoologia, Universidade Federal do Paraná, Curitiba, Brasil.

A identificação dos exemplares de Leptoceridae foi realizada com auxílio de bibliografia especializada (FLINT 1981, 1982, 1983, 1991a, 1991b, 1996; FLINT \& REYES 1991; Holzenthal 1984, 1988, 1989, 1995; HolzentHAl \& MORSE 1996; MERRIT \& CUMMINS 1996) e orientação do Dr. Ralph W. Holzenthal da University of Minnesota, Saint Paul (EUA). Algumas identificações foram confirmadas pelo Dr. Oliver S. Flint Jr. do National Museum of Natural History, Washington, D.C. (EUA).

Os caracteres utilizados na identificação das espécies foram os de genitália masculina. Desta forma, a associação entre machos e fêmeas da mesma espécie só pôde ser realizada para Achoropsyche Holzenthal, 1984, pois este é um gênero monotípico.

Além da falta de conhecimento da diversidade neotropical de tricópteros há ainda a necessidade de revisões taxonômicas em muitos grupos; nesta situação, encontram-se os gêneros Nectopsyche e Oecetis McLachlan, 1877 (WIGgINs 1996; Ralph W. Holzenthal, comunicação pessoal). Para o primeiro não foi possível identificar uma morfoespécie (Nectopsyche sp. 1) e, para o segundo, nenhuma das sete morfoespécies capturadas (Oecetis sp. 1 a sp. 7). 
Para as análises de abundância e sazonalidade foram utilizados apenas os exemplares que puderam ser identificados a nível de espécie, portanto, as sete morfoespécies de Oecetis e uma de Nectopsyche não foram consideradas. Com a finalidade de homogeneizar os dados de captura, estes foram baseados em médias (número de exemplares capturados/número de amostras por coleta).

\section{RESULTADOS E DISCUSSÃO}

\section{Sobre as espécies capturadas}

Os exemplares machos capturados foram identificados em 16 espécies distribuídas nos gêneros Nectopsyche, Oecetis, Achoropsyche e Triplectides Kolenati, 1859 (Tab. I). Os dois primeiros estão representados neste trabalho por sete espécies, enquanto os outros dois por apenas uma espécie cada.

Tabela I. Abundância das espécies de Leptoceridae (exemplares machos) capturadas com armadilha luminosa, em oito localidades do Paraná, durante 13 novilúnios, no período de agosto de 1986 a julho de 1987. (ANT) Antonina, (SJP) Săo José dos Pinhais, (COL) Colombo, (PGR) Ponta Grossa, (TEL) Telêmaco Borba, (JDS) Jundiai do Sul, (GUA) Guarapuava e (FEN) Fênix.

\begin{tabular}{|c|c|c|c|c|c|c|c|c|c|}
\hline Espécies & ANT & SJP & $\mathrm{COL}$ & PGR & TEL & JDS & GUA & FEN & Total \\
\hline \multicolumn{10}{|l|}{ Leptocerinae } \\
\hline \multicolumn{10}{|l|}{ Achoropsychini } \\
\hline Achoropsyche duodecimpunctata & 1 & 0 & 0 & 2 & 16 & 5 & 14 & 302 & 340 \\
\hline \multicolumn{10}{|l|}{ Nectopsychini } \\
\hline Nectopsyche aureovittata & 66 & 0 & 0 & 22 & 59 & 1 & 0 & 8 & 156 \\
\hline Nectopsyche fuscomaculata & 112 & 0 & 0 & 10 & 21 & 0 & 28 & 195 & 366 \\
\hline Nectopsyche muhni & 212 & 0 & 0 & 7 & 51 & 6 & 147 & 535 & 958 \\
\hline Nectopsyche separata & 44 & 1 & 3 & 11 & 2 & 2 & 23 & 4 & 90 \\
\hline Nectopsyche ortizi & 0 & 0 & 0 & 4 & 4 & 0 & 0 & 0 & 8 \\
\hline Nectopsyche sp. 1 & 0 & 2 & 0 & 0 & 0 & 0 & 0 & 0 & 2 \\
\hline Nectopsyche splendida & 0 & 0 & 0 & 1 & 0 & 0 & 0 & 0 & 1 \\
\hline \multicolumn{10}{|l|}{ Oecetini } \\
\hline Oecetis sp. 1 & 4 & 0 & 0 & 1 & 15 & 7 & 1 & 8 & 36 \\
\hline Oecetis sp. 2 & 0 & 0 & 1 & 0 & 0 & 0 & 0 & 0 & 1 \\
\hline Oecetis sp. 3 & 1 & 0 & 0 & 3 & 1 & 1 & 13 & 10 & 29 \\
\hline Oecetis sp 4 & 1 & 0 & 0 & 3 & 0 & 0 & 2 & 4 & 10 \\
\hline Oecetis sp. 5 & 1 & 0 & 0 & 4 & 0 & 0 & 0 & 0 & 5 \\
\hline Oecetis sp. 6 & 1 & 0 & 0 & 2 & 0 & 0 & 0 & 0 & 3 \\
\hline Oecetis sp. 7 & 3 & 0 & 0 & 0 & 1 & 3 & 7 & 2 & 16 \\
\hline \multicolumn{10}{|l|}{ Triplectidinae } \\
\hline \multicolumn{10}{|l|}{ Triplectidini } \\
\hline Triplectides gracilis & 2 & 1 & 0 & 0 & 0 & 1 & 0 & 0 & 4 \\
\hline Total de exemplares & 448 & 4 & 4 & 70 & 170 & 26 & 235 & 1068 & 2025 \\
\hline Total de espécies & 12 & 3 & 2 & 12 & 9 & 8 & 8 & 9 & 16 \\
\hline
\end{tabular}

Nectopsyche é um gênero exclusivo do continente americano, com cerca de 50 espécies descritas. A maior diversidade é encontrada na América do Sul, principalmente na sub-região brasileira (FLINT 1982; HolZENTHAL 1995). No presente trabalho, foram identificadas seis espécies para o gênero: $N$. aureovittata Flint, 1983; N. fuscomaculata Flint, 1983; N. muhni (Návas, 1916); N. ortizi Holzenthal, 1995; N. separata (Banks, 1920) e N. splendida (Návas, 1917), sendo que todas já possuem registro para o Brasil, mas são registradas pela primeira vez para o Paraná. 
Nectopsyche aureovittata e $N$. fuscomaculata possuem praticamente a mesma distribuição geográfica, exceto por haver registros de $N$. fuscomaculata no Amazonas (FLINT 1991a). Ambas possuem registros para Santa Catarina (Brasil) e também para o Chile, Argentina, Paraguai e Uruguai (FLINT 1983).

Nectopsyche muhni distribui-se por grande parte do Brasil: Roraima, Amazonas, Pará, Goiás, Distrito Federal, Espírito Santo, Rio de Janeiro, São Paulo, Santa Catarina e Rio Grande do Sul. Nectopsyche splendida possui distribuição um pouco mais restrita, não tendo sido até o momento citada para a região sul do país: Roraima, Goiás, Espírito Santo e Rio de Janeiro (FLINT 1991a). Estas duas espécies também foram coletadas em trabalho realizado por FLINT (1982) na área do Rio do Prata, Argentina. Nectopsyche splendida é ainda citada em estudo realizado por FLINT (1996) no Parque Manu, Peru.

Nectopsyche ortizi, no Brasil, foi citada somente para o Pará. Também é conhecida no Panamá, México, Costa Rica, Suriname, Venezuela, Guiana, Peru, Paraguai e Argentina (HolzENTHAL 1995).

No Brasil, N. separata foi coletada no Rio de Janeiro e Santa Catarina (FLINT .1972). Na Argentina, foi coletada por FLINT (1982) no levantamento realizado na região do Rio do Prata.

O gênero Oecetis é encontrado em todas as regiões do mundo, sendo muito abundante nas regiões tropicais, onde várias espécies ainda não foram descritas (FLINT 1991b). As sete morfoespécies citadas neste trabalho não puderam ser identificadas e provavelmente representam espécies novas (Ralph W. Holzenthal, comunicação pessoal) e serão descritas oportunamente.

Achoropsyche é um gênero monotípico amplamente distribuído na América do Sul: Colômbia, Venezuela, Guiana, Suriname, Equador, Peru, Brasil (Amazonas, Pará, Espírito Santo e Santa Catarina), Bolívia, Paraguai, Argentina e Uruguai (Flint 1991a; HolzentHal 1984). A espécie A. duodecimpunctata (Návas, 1916), também foi capturada em levantamentos realizados por FLINT (1982) na Argentina, FLINT (1991a) na Ilha de Maracá, Roraima (Brasil) e Flint (1996) no Parque Manu (Peru). Este é o primeiro registro da espécie para o Paraná.

O gênero Triplectides possui cerca de 60 espécies mundialmente distribuídas (HolzENTHAL 1988), sendo 12 reconhecidas para a região Neotropical (FLINT 1991b). Triplectides gracilis (Burmeister, 1839), a única espécie coletada, já havia sido registrada para o Rio de Janeiro (HoLzENTHAL 1988), mas é pela primeira vez citada para o Estado.

MCELRAVy et al. (1981) estudando a diversidade da fauna de tricópteros do Panamá, coletaram três gêneros de Leptoceridae, sendo a espécie Triplectides gracilis a única em comum com as capturadas no PROFAUPAR.

\section{Abundância e sazonalidade}

Um total de 126.340 exemplares de Trichoptera foi capturado com armadilha luminosa durante o primeiro ano do PROFAUPAR (agosto de 1986 a julho de 1987). Destes, 6.375 indivíduos pertencem à família Leptoceridae, sendo que 2.025 exemplares são machos $(31,76 \%)$ e 4.350 fêmeas $(68,24 \%)$ (Tabs II, III). 
Tabela II. Número total de exemplares de Leptoceridae (machos e fêmeas), por gênero, capturados com armadilha luminosa, em oito localidades do Paraná, no periodo de agosto de 1986 a julho de 1987. (ANT) Antonina, (SJP) São José dos Pinhais, (COL) Colombo, (PGR) Ponta Grossa, (TEL) Telêmaco Borba, (JDS) Jundiai do Sul, (GUA) Guarapuava e (FEN) Fênix.

\begin{tabular}{lrrrrrrrrr}
\hline \multicolumn{1}{c}{ Gêneros } & ANT & SJP & COL & PGR & TEL & JDS & GUA & FEN & Total \\
\hline Achoropsyche & 4 & 1 & 0 & 12 & 24 & 21 & 53 & 1028 & 1143 \\
Nectopsyche & 1382 & 13 & 6 & 273 & 690 & 76 & 677 & 1522 & 4639 \\
Oecetis & 120 & 0 & 1 & 64 & 78 & 115 & 148 & 60 & 586 \\
Triplectides & 2 & 3 & 0 & 0 & 0 & 2 & 0 & 0 & 7 \\
\hline Total de exemplares & 1508 & 17 & 7 & 349 & 792 & 214 & 878 & 2610 & 6375 \\
\hline
\end{tabular}

Tabela III. Número de exemplares de Leptoceridae, machos (M) e fêmeas (F), por gênero, capturados com armadilha luminosa, em oito localidades do Paraná, no período de agosto de 1986 a julho de 1987. (ANT) Antonina, (SJP) São José dos Pinhais, (COL) Colombo, (PGR) Ponta Grossa, (TEL) Telêmaco Borba, (JDS) Jundiai do Sul, (GUA) Guarapuava e (FEN) Fênix.

\begin{tabular}{|c|c|c|c|c|c|c|c|c|c|c|c|c|c|c|c|c|c|c|}
\hline \multirow{2}{*}{ Gêneros } & \multicolumn{2}{|c|}{ ANT } & \multicolumn{2}{|c|}{ SJP } & \multicolumn{2}{|c|}{$\mathrm{COL}$} & \multicolumn{2}{|c|}{ PGR } & \multicolumn{2}{|c|}{ TEL } & \multicolumn{2}{|c|}{ JDS } & \multicolumn{2}{|c|}{ GUA } & \multicolumn{2}{|c|}{ FEN } & \multicolumn{2}{|c|}{ Total } \\
\hline & $M$ & $\mathrm{~F}$ & M & $\mathrm{F}$ & M & $\mathrm{F}$ & $M$ & $\mathrm{~F}$ & $M$ & $F$ & $M$ & $\mathrm{~F}$ & M & $\mathrm{F}$ & $M$ & $\mathrm{~F}$ & M & $\mathrm{F}$ \\
\hline Achoropsyche & 1 & 3 & 0 & 1 & 0 & 0 & 2 & 10 & 16 & 8 & 5 & 16 & 14 & 39 & 302 & 26 & 340 & 803 \\
\hline Nectopsyche & 434 & 948 & 3 & 0 & 3 & 3 & 55 & 18 & 137 & 553 & 9 & 67 & 198 & 479 & 742 & 80 & 1581 & 3058 \\
\hline Oecetis & 11 & 109 & 0 & 0 & 1 & 0 & 13 & 51 & 17 & 61 & 11 & 104 & 23 & 125 & 24 & 36 & 100 & 486 \\
\hline Triplectides & 2 & 0 & 1 & 2 & 0 & 0 & 0 & 0 & 0 & 0 & 1 & 1 & 0 & 0 & 0 & 0 & 4 & 3 \\
\hline Total & 448 & 1060 & 4 & 13 & 4 & 3 & 70 & 279 & 170 & 622 & 26 & 188 & 235 & 643 & 1068 & 1542 & 2025 & 4350 \\
\hline
\end{tabular}

Apesar do número de amostras ter sido menor nas localidades de Fênix (57) e Antonina (54), estas apresentaram o maior número de exemplares de Leptoceridae coletados: 2.610 indivíduos (captura média 45,79) e 1.508 (captura média 27,93) respectivamente (Tabs II, IV). Guarapuava com 878 exemplares capturados (captura média 13,51) foi a terceira localidade em número de indivíduos. Telêmaco Borba é a quarta localidade em abundância, com 792 indivíduos (captura média 12,19). Nas demais localidades o número de exemplares capturados foi menor em relação à esta última: Ponta Grossa com 349 indivíduos (captura média 5,36); Jundiai do Sul com 214 (captura média 3,29), São José dos Pinhais com 17 exemplares e Colombo com sete.

Tabela IV. Número médio total de exemplares por gêneros de Leptoceridae (machos e fêmeas) capturados com armadilha luminosa durante 13 novilúnios, em oito localidades do Paraná, no periodo de agosto de 1986 a julho de 1987. (ANT) Antonina, (PGR) Ponta Grossa, (TEL) Telêmaco Borba, (JDS) Jundiaí do Sul, (GUA) Guarapuava e (FEN) Fênix.

\begin{tabular}{lrrrrrrrrr}
\hline \multicolumn{1}{c}{ Generos } & ANT & SJP & COL & PGR & TEL & JDS & GUA & FEN & Total \\
\hline Achoropsyche & 0,07 & 0,02 & 0,00 & 0,18 & 0,37 & 0,32 & 0,82 & 18,04 & 1,52 \\
Nectopsyche & 25,60 & 0,20 & 0,09 & 4,20 & 10,62 & 1,17 & 10,42 & 26,70 & 6,07 \\
Oecetis & 2,22 & 0,00 & 0,02 & 0,98 & 1,20 & 1,77 & 2,27 & 1,05 & 0,73 \\
Triplectides & 0,04 & 0,05 & 0,00 & 0,00 & 0,00 & 0,03 & 0,00 & 0,00 & 0,01 \\
\hline Total de exemplares & 27,93 & 0,27 & 0,11 & 5,36 & 12,19 & 3,29 & 13,51 & 45,79 & 8,33 \\
\hline
\end{tabular}


Comparando esses resultados com aqueles obtidos para a família Hydropsychidae em MARINONI \& ALMEIDA (2000), são observadas modificações na seqüência das localidades com relação à abundância de captura. Em Hydropsychidae, o número de exemplares capturados foi muito maior que o de Leptoceridae, 39.515 indivíduos (seis vezes mais). As localidades que apresentaram maior número de exemplares capturados foram Telêmaco Borba, Guarapuava e Fênix, em seqüência decrescente. Nestes locais, as armadilhas encontravam-se próximas à corpos d'água, o que justificaria a grande quantidade de exemplares, já que estas seriam sítios de oviposição e emergência para os tricópteros.

Relacionar os resultados obtidos com dados biológicos e climáticos torna-se muito difícil, pois pouco se conhece a respeito das espécies de tricópteros neotropicais. As formas imaturas de Leptoceridae habitam os mais variados tipos de ambientes aquáticos, desde rios de grande correnteza a locais quase sem correnteza e de água parada com grande quantidade de matéria orgânica e, conseqüentemente, de pouca oxigenação e com temperatura mais elevada (WIGGINS 1996). A capacidade de completar seu ciclo biológico em locais como pequenos tanques ou até mesmo poças temporárias, pode ser a explicação para o maior número de indivíduos capturados em Antonina. Neste local, a armadilha foi colocada próxima a um pequeno tanque com grande quantidade de matéria orgânica (Fig. 1). Em Fênix, local em que foi capturada a maioria dos indivíduos de Leptoceridae, a armadilha luminosa foi instalada a aproximadamente um quilômetro do rio Corumbataí (Fig. 2) (Renato C. Marinoni, comunicação pessoal).

Três localidades estudadas no PROFAUPAR têm historicamente temperatura média anual bem mais elevada que todas as outras: Jundiaí do Sul, Antonina e Fênix. Segundo MARINONI \& DUTRA (1993) naquele ano (1986/1987) a temperatura média anual em Jundiai foi $22,4^{\circ} \mathrm{C}$ e em Antonina e Fênix, $21,5^{\circ} \mathrm{C}$. Neste mesmo trabalho em uma análise de agrupamento, as localidades de Fênix e Antonina mostraram-se mais assemelhadas em função da variável climatológica temperatura máxima. A maior quantidade de exemplares capturados nestas duas últimas localidades é provavelmente resultado da associação entre a proximidade das armadilhas aos corpos d'água e temperatura ambiente mais elevada.

Em todas as localidades estudadas, com exceção de Colombo, o número total de fềmeas capturadas foi maior que o de machos (Tabs III, V). Este fato também foi observado para a família Hydropsychidae (Kovats et al. 1996; Flint et al. 1987; MARINONI \& ALMEIDA 2000). Segundo estes autores, a maior captura de fêmeas pode estar relacionada ao comportamento reprodutivo das espécies e à maior atração destas pela luz. Para Leptoceridae, no entanto, nenhuma observação ou estudo que corroborem estas hipóteses foram realizados até o momento.

Dos quatro gêneros de Leptoceridae coletados, o que teve maior representatividade foi Nectopsyche com $72,77 \%$ do total capturado (4.639 indivíduos) (Tab. II). Este foi também o único com representantes em todas as localidades. O número de fêmeas capturadas foi, de modo geral, maior nas seguintes proporções: em Fênix 1 macho para 1,05 fêmeas; em Antonina 1:2,2; em Guarapuava 1:2,4; em Telêmaco Borba 1:4; em Ponta Grossa 1:5; em Jundiaí do Sul 1:7,4; em São José dos Pinhais 1:3,3 e em Colombo 1:1 (Tab. V). 


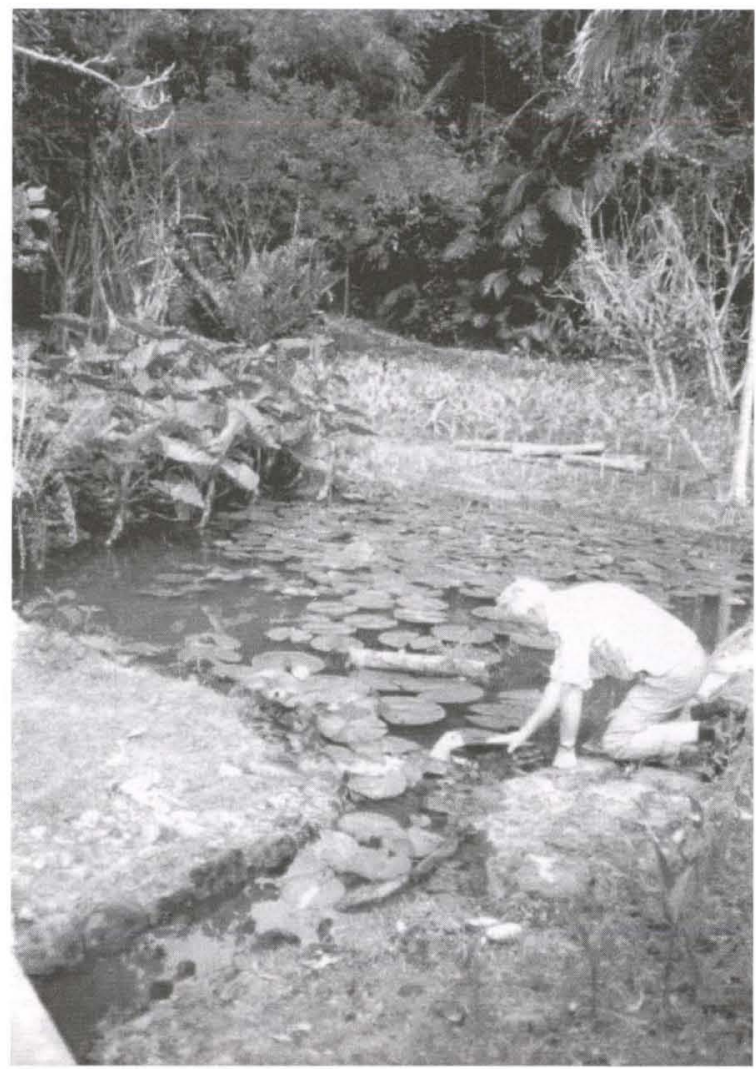

Fig. 1. Local de instalação da armadilha luminosa em Antonina.

Tabela V. Proporção de machos (M) e fêmeas $(F)$ por gêneros, capturados com armadilha luminosa durante 13 novilúnios, em oito localidades do Paraná, no periodo de agosto de 1986 a julho de 1987. (ANT) Antonina, (PGR) Ponta Grossa, (TEL) Telêmaco Borba, (JDS) Jundiai do Sul, (GUA) Guarapuava e (FEN) Fênix.

\begin{tabular}{|c|c|c|c|c|c|c|c|c|c|}
\hline Gêneros & ANT & SJP & $\mathrm{COL}$ & PGR & TEL & JDS & GUA & FEN & Total \\
\hline Achoropsyche & $1: 3$ & $0: 1$ & - & $1: 5$ & $2: 1$ & $1: 3,2$ & $1: 2,8$ & $1: 24$ & $1: 2,3$ \\
\hline Nectopsyche & $1: 2,2$ & $1: 3,3$ & $1: 1$ & $1: 5$ & $1: 4$ & $1: 7,4$ & $1: 2,4$ & $1: 1,05$ & $1: 1,9$ \\
\hline Oecetis & $1: 9,9$ & - & $1: 0$ & $1: 3,9$ & $1: 3,6$ & $1: 9,4$ & $1: 5,4$ & $1: 1,5$ & $1: 4,8$ \\
\hline Triplectides & $2: 0$ & $2: 1$ & - & - & - & $1: 1$ & - & - & $1,33: 1$ \\
\hline Total de exemplares & $1: 2,4$ & $1: 3,25$ & $1,33: 1$ & $1: 3,98$ & $1: 3,66$ & $1: 7,23$ & $1: 2,74$ & $1: 1,44$ & \\
\hline
\end{tabular}

Em relação à sazonalidade de machos e fêmeas algumas diferenças foram observadas para o gênero Nectopsyche. Em Ponta Grossa, somente fêmeas foram capturadas em agosto e fevereiro. (Fig. 3). Em Jundiai do Sul em março, abril e julho só houve captura de fêmeas (Fig. 4). Em Guarapuava apenas exemplares fêmeas foram capturadas em setembro e abril; nos outros meses, a abundância destas 


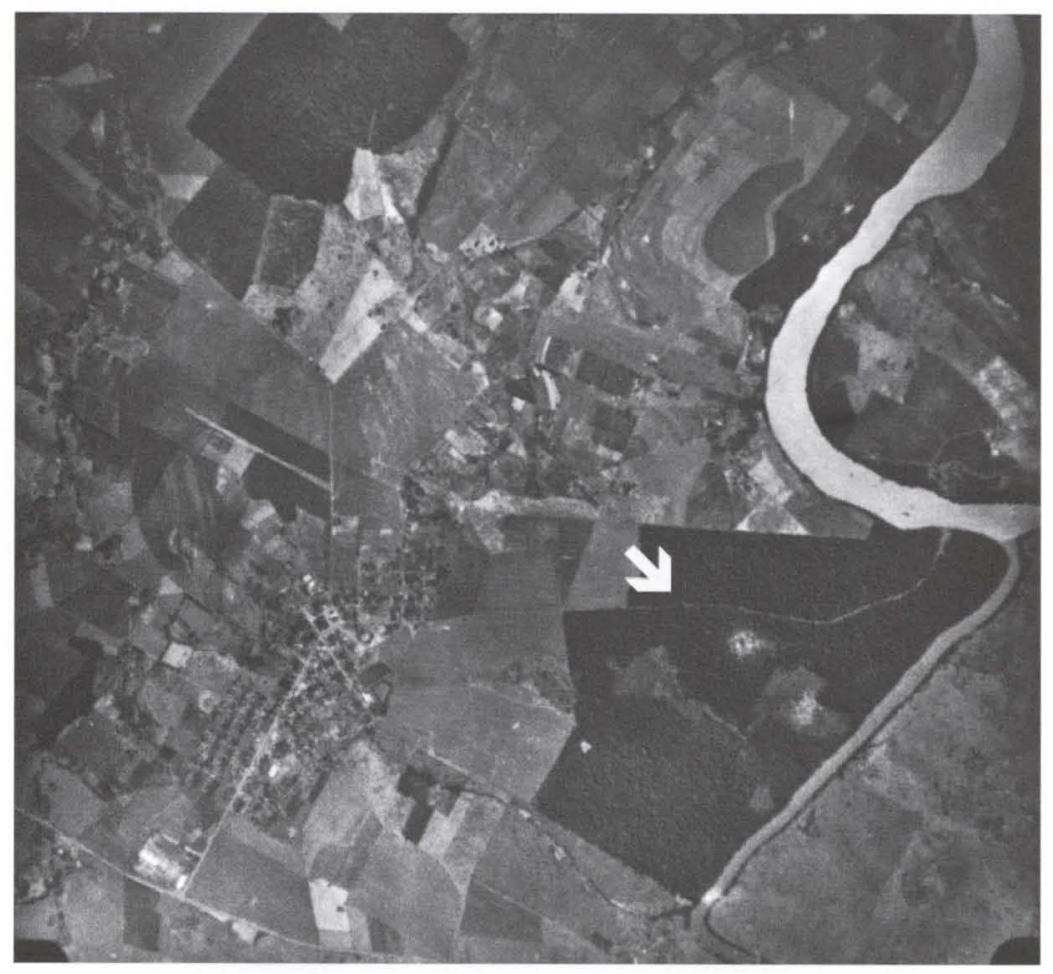

Fig. 2. Vista aérea de Fênix. A seta branca indica o local em que a armadilha foi instalada.

foi sempre maior (Fig. 5). Em Telêmaco Borba somente fêmeas foram capturadas em abril e machos em julho; nenhum exemplar foi capturado em agosto, setembro, maio e junho (Fig. 6).

Analisando-se os gráficos, observa-se que em todas as localidades a sazonalidade de Nectopsyche parece ter sido influenciada pela variável temperatura mínima (Figs 3-8). No entanto, como já mencionado anteriormente, as localidades de Fênix e Antonina estavam mais relacionadas pela variável temperatura máxima (MARINONI \& DUTRA 1993). Isto fica bastante evidente em Antonina, onde a relação da distribuição dos exemplares ao longo do ano com a variação das médias das temperaturas nos dias de coleta é nítida (Fig. 7). Assim, para estas localidades foi adicionada ao gráfico a curva que representa esta variável durante aquele ano.

Tanto em Fênix como em Antonina, a sazonalidade e a abundância do gênero foram mais uniformes; entretanto, os picos de captura são diferentes. Em Fênix muitos exemplares foram capturados em meses que geralmente têm temperaturas mais baixas (julho e agosto), além de grande abundância em novembro, janeiro e março (Fig. 8). É interessante ressaltar que todas as espécies capturadas em Antonina, inclusive aquelas de Hydropsychidae (MARINONI \& ALMEIDA 2000), tiveram picos de ocorrência em março (Figs 10, 13, 14). Em Guarapuava, nos meses de setembro, maio e junho, houve uma queda bastante significativa da temperatura, 

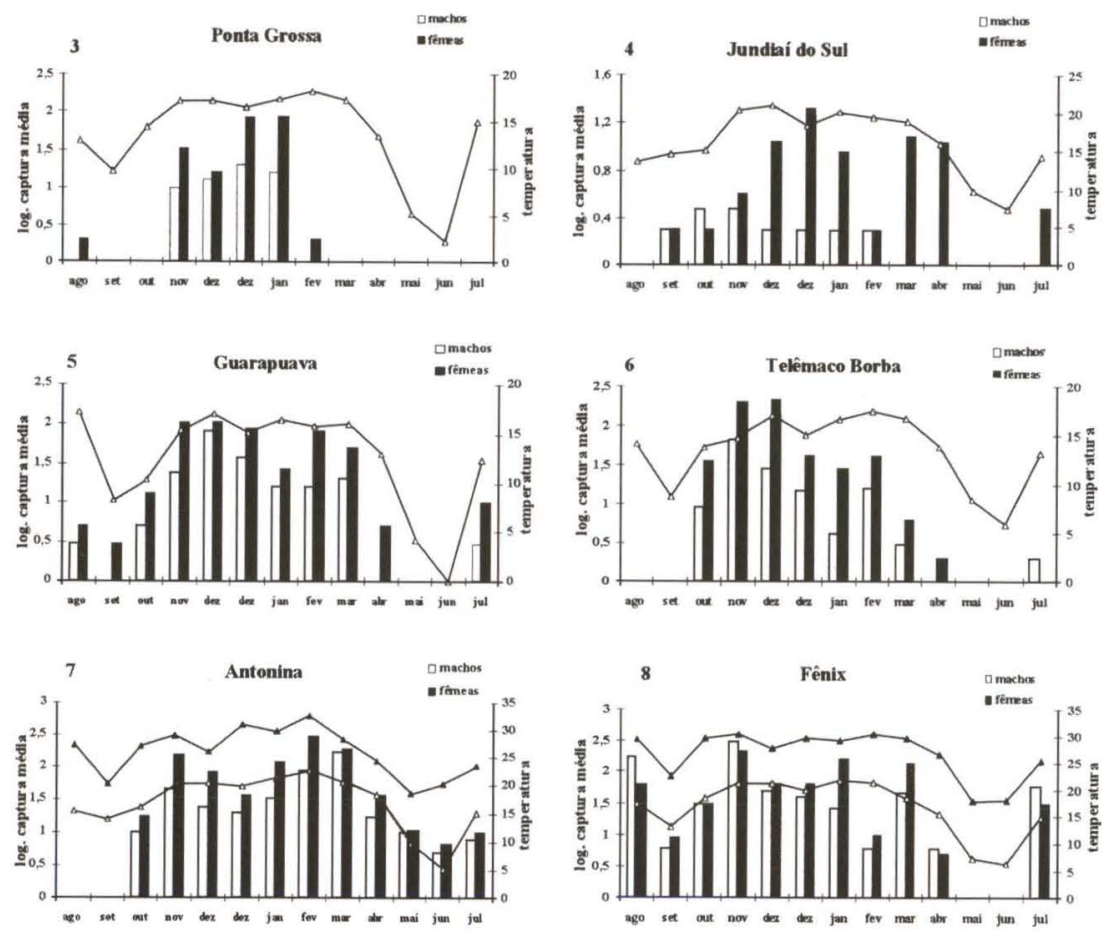

Figs 3-8. Número médio de exemplares machos e fêmeas (valores em logarítmo, $n+1$ ) de Nectopsyche, capturados com armadilha luminosa durante treze novilúnios em seis localidades do Paraná e os valores das variáveis temperatura minima $(\mathbf{\Delta})$ e máxima $(\Delta)$ em graus Celsius.

chegando a graus negativos nos dois últimos (Fig. 5). Nestes meses, como em Hydropsychidae, nenhuma espécie foi capturada (MARINONI \& ALMEIDA 2000).

Nectopsyche muhni foi a espécie com o maior número médio de exemplares machos capturados $(15,3)$. As localidades que tiveram maior captura média foram Fênix com 8,75 exemplares (55,84\%), Antonina com 3,29 indivíduos $(22,12 \%)$ e Guarapuava com 2,26 espécimes (14,77\%) (Tab. VI). A maior captura média de $N$. muhni em Fênix ocorreu no mês de novembro $(61,6)$, enquanto que em Antonina os maiores picos foram obtidos em fevereiro e março (10,4 e 17,8 respectivamente) (Figs 9, 10). Em Guarapuava o pico ocorreu no primeiro novilúnio de dezembro $(14,0)$ (Fig. 11).

A segunda espécie com maior captura média de exemplares machos foi Nectopsyche fuscomaculata, com 5,65 individuos. Destes, 3,00 (53,27\%) em Fênix e 1,75 (30,60\%) em Antonina (Tab. VI). Como se observa na figura 12, em Fênix, a espécie foi mais abundante nos meses de agosto e julho, sendo sua sazonalidade responsável pelo pico de captura do gênero em meses de temperatura mais baixa. $\mathrm{Na}$ localidade de Antonina a maior captura foi obtida em março (Fig. 13). 

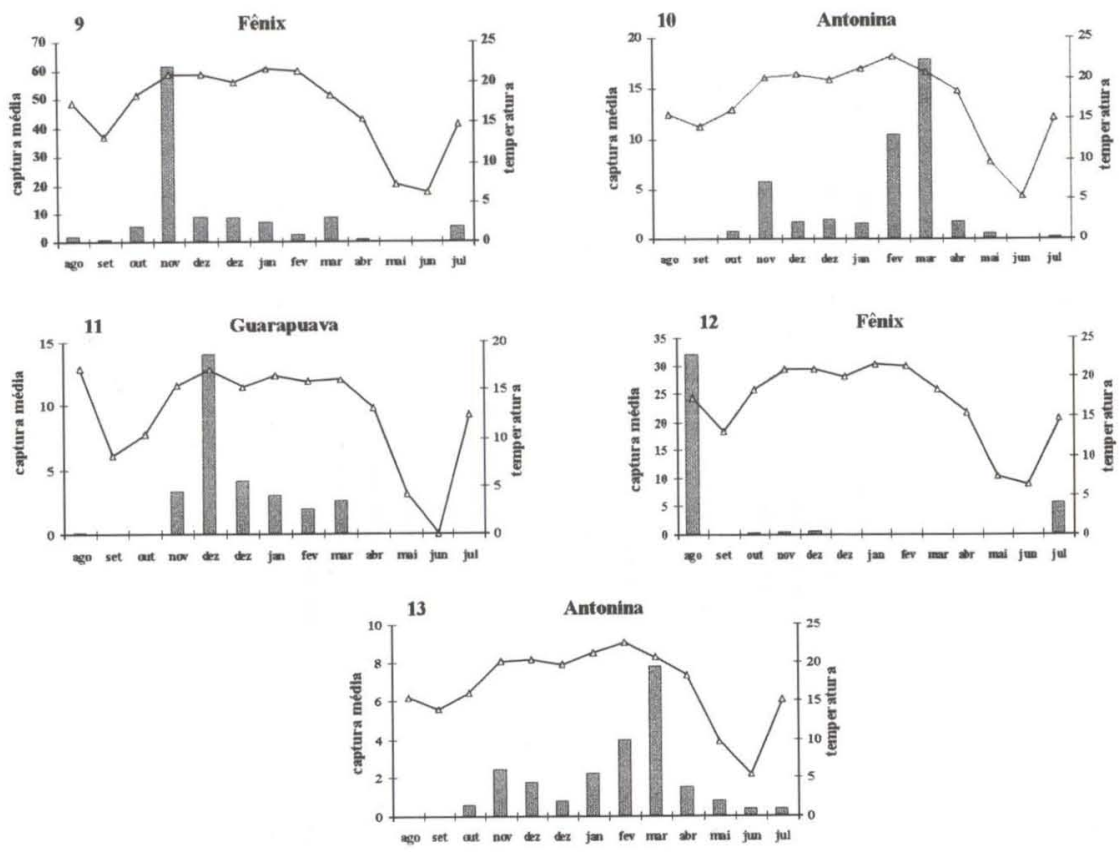

Figs 9-13. Número médio de exemplares machos capturados com armadilha luminosa durante treze novilúnios em três localidades do Paraná. (9-11) Nectopsyche muhni; (12-13) Nectopsyche fuscomaculata. Temperatura minima $(\mathbf{\Delta})$ em graus Celsius.
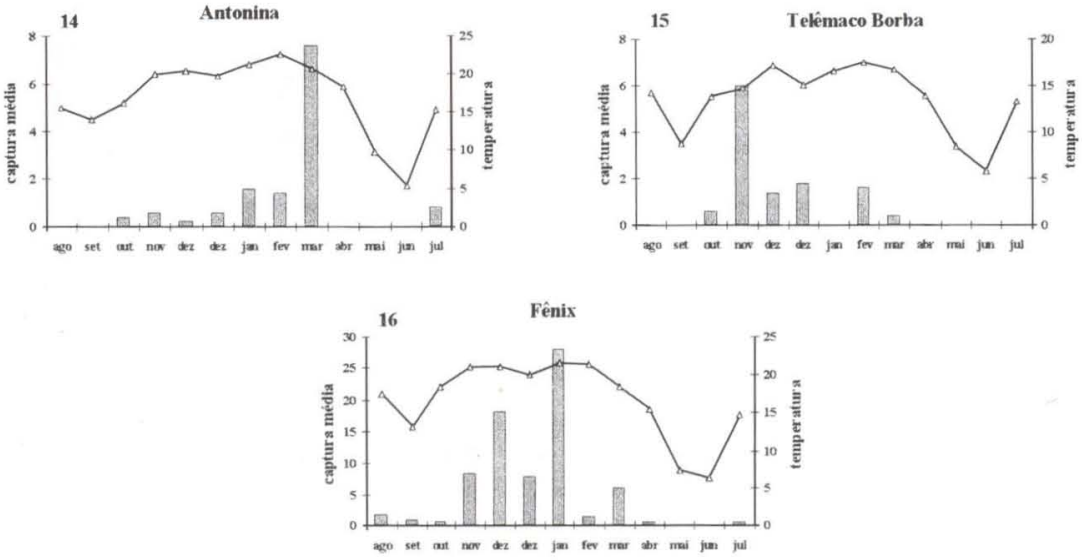

Figs 14-16. Número médio de exemplares capturados com armadilha luminosa durante treze novilúnios em três localidades do Paraná. (14-15) Nectopsyche aureovittata (exemplares machos); (16) Achoropsyche duodecimpunctata (exemplares machos e fêmeas). Temperatura minima $(\boldsymbol{\Delta})$ em graus Celsius. 
Tabela VI. Número médio mensal das espécies de Leptoceridae (exemplares machos) capturadas com armadilha luminosa durante 13 novilúnios, em oito localidades do Paraná, no período de agosto de 1986 a julho de 1987. Para Achoropsyche duodecimpunctata foram somados exemplares machos e fêmeas.

\begin{tabular}{|c|c|c|c|c|c|c|c|c|c|c|c|c|c|c|}
\hline Local & Ago & Set & Out & Nov & Dez & Dez & Jan & Fev & Mar & $\mathrm{Abr}$ & Mai & Jun & Jul & Total \\
\hline \multicolumn{15}{|l|}{ Fênix } \\
\hline A. duodecimpunctata & 1,8 & 1,0 & 0,6 & 8,4 & 17,2 & 8,0 & 28,0 & 1,5 & 6,0 & 0,5 & 0,0 & 0,0 & 0,4 & 5,65 \\
\hline N. aureovittata & 0,0 & 0,0 & 0,0 & 0,2 & 0,0 & 1,0 & 1,0 & 0,0 & 0,0 & 0,0 & 0,0 & 0,0 & 0,0 & 0,17 \\
\hline N. fuscomaculata & 32,2 & 0,0 & 0,2 & 0,4 & 0,6 & 0,0 & 0,0 & 0,0 & 0,0 & 0,0 & 0,0 & 0,0 & 5,6 & 3,00 \\
\hline N. muhni & 2,0 & 1,3 & 5,8 & 61,6 & 9.0 & 8,8 & 7,3 & 2,5 & 8,8 & 1,3 & 0,0 & 0,0 & 5,4 & 8,75 \\
\hline N. separata & 0,0 & 0,0 & 0,0 & 0,0 & 0,0 & 0,0 & 0,0 & 0,0 & 0,0 & 0,0 & 0,0 & 0,0 & 0,8 & 0,06 \\
\hline Total & 36,0 & 2,3 & 6,6 & 70,6 & 26,8 & 17,8 & 36,3 & 4,0 & 14,8 & 1,8 & 0.0 & 0,0 & 12,2 & 17,63 \\
\hline \multicolumn{15}{|l|}{ Antonina } \\
\hline A. duodecimpunctata & 0,0 & 0,0 & 0,0 & 0,0 & 0.0 & 0,0 & 0,2 & 0,0 & 0,0 & 0,0 & 0,0 & 0,0 & 0,0 & 0,02 \\
\hline N. aureovittata & 0,0 & 0,0 & 0,4 & 0,6 & 0,2 & 0,6 & 1,6 & 1,4 & 7,6 & 0,0 & 0,0 & 0,0 & 0,8 & 1,02 \\
\hline N. fuscomaculata & 0,0 & 0,0 & 0,6 & 2,4 & 1,8 & 0,8 & 2,2 & 4,0 & 7,8 & 1,5 & 0,8 & 0,4 & 0,4 & 1,75 \\
\hline N. muhni & 0,0 & 0,0 & 0,8 & 5,8 & 1,8 & 2,0 & 1,6 & 10,4 & 17,8 & 1,8 & 0,6 & 0,0 & 0,2 & 3,29 \\
\hline N. separata & 0,0 & 0,0 & 0,0 & 0,2 & 0,8 & 0,4 & 0,6 & 1,2 & 4,2 & 0.8 & 0,4 & 0,4 & 0,0 & 0,69 \\
\hline T. gracilis & 0,0 & 0,0 & 0,2 & 0,2 & 0,0 & 0,0 & 0,0 & 0,0 & 0,0 & 0,0 & 0.0 & 0,0 & 0,0 & 0,03 \\
\hline Total & 0,0 & 0,0 & 2,0 & 9,2 & 4,6 & 3,8 & 6,2 & 17,0 & 37,4 & 4,1 & 1,8 & 0,8 & 1,4 & 6,80 \\
\hline \multicolumn{15}{|l|}{ Guarapuava } \\
\hline A. duodecimpunctata & 0,0 & 0.0 & 0,2 & 0,4 & 0,8 & 0,8 & 0,2 & 0,0 & 0,0 & 0,0 & 0,0 & 0,0 & 0,0 & 0,22 \\
\hline N. fuscomaculata & 0.2 & 0.0 & 0,4 & 0,6 & 1,4 & 1,4 & 0,0 & 1,0 & 0,4 & 0,0 & 0.0 & 0,0 & 0,2 & 0,43 \\
\hline N. muhni & 0,2 & 0,0 & 0,0 & 3,4 & 14,0 & 4,2 & 3,0 & 2,0 & 2,6 & 0,0 & 0.0 & 0,0 & 0,0 & 2,26 \\
\hline N. separata & 0,0 & 0,0 & 0,4 & 0,6 & 1,0 & 1,6 & 0,0 & 0,0 & 0,8 & 0,0 & 0,0 & 0,0 & 0,2 & 0,35 \\
\hline Total & 0,4 & 0,0 & 1,0 & 5,0 & 17,2 & 8,0 & 3,2 & 3,0 & 4,2 & 0,0 & 0.0 & 0,0 & 0,4 & 3,26 \\
\hline \multicolumn{15}{|l|}{ Telêmaco Borba } \\
\hline A. duodecimpunctata & 0,0 & 0,0 & 0,0 & 0,0 & 1,2 & 1,8 & 0,0 & 0,2 & 0,0 & 0,0 & 0,0 & 0,0 & 0,0 & 0,25 \\
\hline N. aureovittata & 0,0 & 0,0 & 0,6 & 6,0 & 1,4 & 1,8 & 0,0 & 1,6 & 0,4 & 0,0 & 0.0 & 0,0 & 0,0 & 0,91 \\
\hline N. fuscomaculata & 0,0 & 0,0 & 0,0 & 1,8 & 1,4 & 0,0 & 0,4 & 0,4 & 0,0 & 0,0 & 0,0 & 0,0 & 0,2 & 0,32 \\
\hline N. muhni & 0,0 & 0,0 & 1,0 & 5,6 & 2,4 & 0,8 & 0,0 & 0,6 & 0,0 & 0,0 & 0,0 & 0,0 & 0,0 & 0,80 \\
\hline N. separata & 0,0 & 0,0 & 0,0 & 0,0 & 0,4 & 0,0 & 0,0 & 0,0 & 0,0 & 0,0 & 0,0 & 0,0 & 0,0 & 0,03 \\
\hline N. ortizi & 0,0 & 0,0 & 0,0 & 0,0 & 0,0 & 0,2 & 0,2 & 0,4 & 0,0 & 0,0 & 0,0 & 0,0 & 0,0 & 0,06 \\
\hline Total & 0,0 & 0,0 & 1,6 & 13,4 & 6,8 & 4,6 & 0,6 & 3,2 & 0,4 & 0,0 & 0,0 & 0,0 & 0,2 & 2,37 \\
\hline \multicolumn{15}{|l|}{ Ponta Grossa } \\
\hline A. duodecimpunctata & 0,0 & 0,0 & 0,0 & 0,0 & 0,0 & 0,2 & 0,2 & 0,0 & 0,0 & 0,0 & 0,0 & 0,0 & 0,0 & 0,03 \\
\hline N. aureovittata & 0,0 & 0,0 & 0,0 & 1,4 & 2,2 & 0,0 & 0,8 & 0,0 & 0,0 & 0,0 & 0,0 & 0,0 & 0,0 & 0,34 \\
\hline N. fuscomaculata & 0,0 & 0,0 & 0,0 & 0,0 & 0,0 & 1,0 & 1,0 & 0,0 & 0,0 & 0,0 & 0,0 & 0,0 & 0,0 & 0,15 \\
\hline N. muhni & 0,0 & 0,0 & 0,0 & 0,4 & 0,0 & 0,8 & 0,2 & 0,0 & 0,0 & 0,0 & 0,0 & 0,0 & 0,0 & 0,11 \\
\hline N. separata & 0,0 & 0,0 & 0,0 & 0,0 & 0,0 & 1,8 & 0,4 & 0,0 & 0,0 & 0,0 & 0,0 & 0,0 & 0,0 & 0,17 \\
\hline N. ortizi & 0,0 & 0,0 & 0,0 & 0,0 & 0,2 & 0,0 & 0,6 & 0,0 & 0,0 & 0,0 & 0,0 & 0,0 & 0,0 & 0,06 \\
\hline N. splendida & 0,0 & 0,0 & 0,0 & 0,0 & 0,0 & 0,2 & 0,0 & 0,0 & 0,0 & 0,0 & 0,0 & 0,0 & 0,0 & 0,02 \\
\hline Total & 0,0 & 0,0 & 0,0 & 1,8 & 2,4 & 4,0 & 3,2 & 0,0 & 0,0 & 0,0 & 0,0 & 0,0 & 0,0 & 0,88 \\
\hline \multicolumn{15}{|l|}{ Jundiai do Sul } \\
\hline A. duodecimpun & 0,0 & 0,0 & 0,0 & 0,2 & 0,8 & 0,0 & 0,0 & 0,0 & 0,0 & 0,0 & 0.0 & 0,0 & 0,0 & 0,08 \\
\hline N. aureovittata & 0,0 & 0,2 & 0,0 & 0,0 & 0.0 & 0,0 & 0,0 & 0,0 & 0,0 & 0,0 & 0.0 & 0,0 & 0,0 & 0,02 \\
\hline N. muhni & 0,0 & 0,0 & 0,4 & 0,4 & 0,2 & 0,2 & 0,0 & 0,0 & 0,0 & 0,0 & 0,0 & 0,0 & 0,0 & 0,09 \\
\hline N. separata & 0,0 & 0,0 & 0,0 & 0,0 & 0,0 & 0,0 & 0,2 & 0,2 & 0,0 & 0,0 & 0,0 & 0,0 & 0,0 & 0,03 \\
\hline T. gracilis & 0,0 & 0,0 & 0,2 & 0,0 & 0,0 & 0,0 & 0,0 & 0,0 & 0,0 & 0,0 & 0,0 & 0,0 & 0,0 & 0,02 \\
\hline Total & 0,0 & 0,2 & 0,6 & 0,6 & 1,0 & 0,2 & 0,2 & 0,2 & 0,0 & 0.0 & 0,0 & 0,0 & 0,0 & 0,24 \\
\hline \multicolumn{15}{|l|}{ Săo José dos Pinhais } \\
\hline N. separata & 0,0 & 0,0 & 0,0 & 0,0 & 0,0 & 0,2 & 0,0 & 0,0 & 0,0 & 0,0 & 0,0 & 0,0 & 0,0 & 0,02 \\
\hline T. gracilis & 0,0 & 0,0 & 0,0 & 0,2 & 0,0 & 0,0 & 0,0 & 0,0 & 0,0 & 0,0 & 0,0 & 0,0 & 0,0 & 0,02 \\
\hline Total & 0,0 & 0,0 & 0,0 & 0,2 & 0,0 & 0,2 & 0,0 & 0.0 & 0,0 & 0,0 & 0,0 & 0,0 & 0,0 & 0,04 \\
\hline \multicolumn{15}{|l|}{ Colombo } \\
\hline N. separata & 0,0 & 0,0 & 0,0 & 0,0 & 0,0 & 0,2 & 0,0 & 0,0 & 0,2 & 0,2 & 0,0 & 0,0 & 0,0 & 0,05 \\
\hline otal & 0,0 & 0,0 & 0,0 & 0,0 & 0,0 & 0,2 & 0,0 & 0,0 & 0,2 & 0,2 & 0,0 & 0,0 & 0,0 & 0,05 \\
\hline
\end{tabular}


Nectopsyche aureovittata, a quarta em abundância, teve 2,46 indivíduos capturados; 1,02 (42,30\%) em Antonina e 0,91 (37,82\%) em Telêmaco Borba (Tab. VI). Como observado para as espécies citadas anteriormente, $N$. aureovittata também teve maior número de exemplares capturados no mês de março em Antonina. Já em Telêmaco Borba, a espécie foi mais abundante em novembro (Figs 14, 15).

A espécie Nectopsyche separata, apesar de não ser a mais abundante foi a única a ser capturada em todas as oito localidades estudadas. Já Nectopsyche ortizi foi capturada somente em Ponta Grossa e Telêmaco Borba, quatro exemplares em cada uma destas localidades (Tabs I, VI). Apenas uma espécie não pôde ser identificada e foi designada Nectopsyche sp. 1. Esta teve apenas dois exemplares capturados em São José dos Pinhais.

Ao contrário do que ocorreu com Nectopsyche, a associação de machos e fêmeas em Achoropsyche foi possível por se tratar de um gênero monotípico. No entanto, a biologia e a ecologia da espécie é ainda pouco estudada. Os adultos são comumente coletados nas proximidades de grandes rios, mas nada a respeito do comportamento das formas imaturas é conhecido (HOLZENTHAL 1984).

Achoropsyche duodecimpunctata é a terceira espécie com o maior número de exemplares capturados (6,25), sendo $89,94 \%$ dos indivíduos capturados na localidade de Fênix (Tabs II, VI). O maior número de exemplares foi capturado em janeiro (Fig. 16).

Apesar do maior número de exemplares capturados em Fênix, esta localidade não foi a que apresentou maior número de espécies. As localidades de Antonina e Ponta Grossa foram as que tiveram maior riqueza: 12 espécies cada. Seguidas por Fênix e Telêmaco Borba, com nove espécies. Guarapuava e Jundiaí do Sul tiveram oito espécies capturadas e por último, São José e Colombo com três e duas espécies respectivamente.

AGRADECIMENTOS. Especialmente ao Dr. Ralph W. Holzenthal (University of Minnesota, EUA) pela orientação e auxílio na identificação das espécies e ao Dr. Renato C. Marinoni pela orientação na análise dos dados e empréstimo das fotografias. Ao Dr. Oliver S. Flint Jr. (National Museum of Natural History, Washington, D.C., EUA) pela confirmação da identificação de algumas espécies. Ao MSc. Marcelo Duarte pela leitura crítica e sugestões.

\section{REFERÊNCIAS BIBLIOGRÁFICAS}

Angrisano, E.B. 1995. Insecta Trichoptera, p. 1199-1237. In: E.C. Lopretto \& G. Tell. Ecossistemas de aguas continentales. Metodologias para su estudio. La Plata, Argentina, Edicones Sur, 1401p. FLINT JR., O.S. 1972. Studies of Neotropical caddisflies, XIV: On a collection from Northern Argentina.

Proc. Biol. Soc. Wash. 85 (17): 223-248.

- 1981. Studies of Neotropical caddisflies, XXVIII: The Trichoptera of the Río Limón Basin, Venezuela. Smithson. Contrib. Zool. 330: 1-60. 1982. Trichoptera of Area Platense. Biol. Acuatica 2: 1-70.

1983. Studies of Neotropical caddisflies, XXXII: New species from Austral South America (Trichoptera). Smithson. Contrib. Zool. 377: 1-100.

- 1991a. Studies of Neotropical caddisflies: on a collection from Ilha de Maracá, Brazil. Acta Amazonica 21: 63-83. 
1991b. Studies of Neotropical caddisflies, XLV: The taxonomy, phenology, and faunistics of the Trichoptera of Antioquia, Colombia. Smithson. Contrib. Zool. 520: 1-113.

1996. The Trichoptera collected on the expeditions to Parque Manu, Madre de Dios, Peru, p. 369-430. In: D.E. Wilson \& A. SAndoval (Eds). Manu. The Biodiversity of Southeastern Peru. La Biodiversidad del Sureste del Peru. Washington, D.C., Smithisonian Inst. Press, 679p. FLint JR., O.S.; J.F. MCAlPine \& H.H. Ross. 1987. A revision of the genus Leptonema Guérin (Trichoptera: Hydropsychidae: Macronematinae). Smithson. Contrib. Zool. 450: 1-193.

FLINT JR., O.S. \& L.A. REYES. 1991. Studies of Neotropical caddisflies, XLVI: The Trichoptera of the Río Moche Basin, Department of la Libertad, Peru. Proceedings of the Biological Society of Washington. Proc. Biol. Soc. Wash. 104 (3): 474-492.

Holzenthal, R.W. 1984. Studies in Neotropical Leptoceridae (Trichoptera) I: Achoropsyche, a new genus. Ann. Entomol. Soc. Amer. 81: 187-208.

1988. Systematics of Neotropical Triplectides (Trichoptera: Leptoceridae). Ann. Entomol. Soc. Amer. 81: 187-208.

1989. Studies in Neotropical Leptoceridae (Trichoptera) IX: A new genus and species from Southeastern Brazil. Aquat. Insects 11: 29-32.

. 1995. The caddisfly genus Nectopsyche: new group species from Costa Rica and the Neotropics (Trichoptera: Leptoceridae). Jour. North Amer. Benthol. Soc. 14 (1): 61-83.

Holzenthal, R.W. \& J.C. MoRse. 1996. Trichoptera Genera, p. 350-386. In: R.W. MERRIT \& K.W. Cummins (Eds). An Introduction to the Aquatic Insects of North America. Dubuque, Kendall/Hunt, XIII $+862 p$.

Kovats, Z.E.; J.J. Ciborowiski \& L.D. CORKUM. 1996. Inland dispersal of adult aquatic insects. Freshwater Biol. 36 (92): 265-276.

Marinoni, L. \& G.L. AlmEida. 2000. Abundância e sazonalidade das espécies de Hydropsychidae (Insecta, Trichoptera) capturadas em armadilha luminosa no estado do Paraná, Brasil. Revta bras. Zool. 17 (1): 283-299.

Marinoni, R.C. \& R.R.C. Dutra. 1993. Levantamento da fauna entomológica no Estado do Paraná. I. Introdução. Situações climáticas e florísticas de oito pontos de coleta. Dados faunísticos de agosto de 1986 a julho de 1987. Revta bras. Zool. 8 (1/2/3/4): 31-73.

MCElravy, E.P.; V.H. ReSH; H. WOLDA \& O.S. Flint JR. 1981. Diversity of adult Trichoptera in a non-seasonal tropical environment. Proc. $3^{\text {rd }}$ Int. Symp. Trichoptera 20: 149-156.

Merrit, R.W. \& K.W. Cummins. 1996. An Introduction to the Aquatic Insects of North America. Dubuque, Kendall/Hunt, 3rd ed., 862p.

PÉREZ, G.R. 1988. Guia para el estudio de los macroinvertebrados acuáticos del Departamento de Antioquia. Bogotá, Colombia, Fondo Fen Colombia/Colciencias/Universidad de Antioquia, 217p.

Wiggins, G.B. 1996. Larvae of the North American Caddisfly Genera (Trichoptera). Toronto, University of Toronto Press, $2^{\text {nd }}$ ed., 401 p.

Recebido em 09.VI.1999; aceito em 05.IV.2000. 